\title{
Nuevo enfoque en la prevención del ictus en pacientes con fibrilación auricular no valvular en hemodiálisis: cierre percutáneo de orejuela izquierda
}

\author{
New approach to the prevention of stroke in patients with non-valvular fibrillation \\ in hemodialysis: percutaneous closure of left atrial appendage
}

\author{
Francisco Torres-Saura' ${ }^{1,2 *}$, Manuela Romero-Vazquiánez ${ }^{1,2}$, Patricio Pérez-Berbel', \\ Eva Cotilla-de la Rosa ${ }^{3}$, Beatriz Diez-Ojea ${ }^{4}$ y Eduardo Arroyo-Úcar ${ }^{1,2}$ \\ ${ }^{1}$ Servicio de Cardiología, Hospital Universitario Vinalopó; '2Servicio de Cardiología, Hospital Universitario de Torrevieja; ${ }^{3}$ Servicio de Nefrología, \\ Hospital Universitario Vinalopó; ${ }^{4}$ Servicio de Nefrología, Hospital Universitario de Torrevieja, Alicante, España
}

Señor editor:

La prevención de episodios cardioembólicos en pacientes con fibrilación auricular (FA) y alto riesgo hemorrágico supone un reto terapéutico, en la medida en la que debe valorarse la conveniencia o no de anticoagular frente al riesgo de sangrado.

Los pacientes con enfermedad renal crónica terminal (ERCT) tienen mayor prevalencia de FA que la población general (entre $12 \%$ y $27 \%$ ), mayor tendencia a la hipercoagulabilidad y fenómenos trombóticos y mayor riesgo de hemorragia por alteraciones de la hemostasia primaria (disfunción plaquetaria) 1 .

Los compuestos más usados como tratamiento anticoagulante en pacientes nefrópatas son los fármacos antivitamina K (warfarina y acenocumarol). En pacientes con ERCT su uso es controvertido, no sólo por el aumento del riesgo de sangrado, sino por las dificultades para mantener un INR en sus límites², la calcificación tisular, la calcifilaxia y el aumento de la arteriosclerosis, así como el mayor riesgo de hospitalizaciones de causa cardiovascular ${ }^{3}$. Además, el uso de los nuevos anticoagulantes de acción directa es limitado porque no hay evidencia científica que sustente su eficacia, ya que los pacientes con ERCT se excluyeron de los estudios clínicos que han demostrado beneficio de éstos respecto de la warfarina ${ }^{4}$.

Por todo lo anterior, los pacientes con ERCT representan un escenario atractivo en el cual el cierre de la orejuela izquierda (COI) puede tener un beneficio clínico claro.

Seis pacientes con ERCT en programa de hemodiálisis, con diagnóstico de FA y problemas con la anticoagulación oral (ACO), se seleccionaron de forma conjunta entre los Servicios de Nefrología y Cardiología para COI, entre junio de 2017 y diciembre de 2018. Todos los pacientes otorgaron su consentimiento por escrito. Los criterios de exclusión fueron tener indicación de ACO por otra causa distinta de la FA, derrame pericárdico grave, cierre previo del tabique interauricular, trombo intracardíaco, hepatopatía crónica grave y el rechazo expreso del paciente. Las características clínicas de los individuos seleccionados se exponen en la tabla 1.

El dispositivo utilizado en todos los casos fue el Watchman ${ }^{\circledR}$ (Boston Scientific Corporation, Marlborough, Massachusetts, EE.UU.). El implante tuvo éxito en todos los casos y no se registraron complicaciones relacionadas ni con el dispositivo ni con el procedimiento.

\section{Correspondencia:}

*Francisco Torres-Saura

E-mail: ftorressaura@gmail.com
Fecha de recepción: 16-04-2019

Fecha de aceptación: 08-07-2019 DOI: 10.24875/ACM.19000190
Disponible en internet: 30-01-2020 Arch Cardiol Mex. 2020;90(1):102-105 www.archivoscardiologia.com 2604-7063/@ 2019 Instituto Nacional de Cardiología Ignacio Chávez. Publicado por Permanyer. Este es un artículo open access bajo la licencia CC BY-NC-ND (http://creativecommons.org/licenses/by-nc-nd/4.0/). 
Tabla 1. Características clínicas de los pacientes incluidos y control a los tres a seis meses de la intervención

\begin{tabular}{|c|c|c|c|c|c|c|}
\hline Paciente & 1 & 2 & 3 & 4 & 5 & 6 \\
\hline Sexo & Hombre & Hombre & Mujer & Hombre & Mujer & Hombre \\
\hline Edad & 71 & 78 & 47 & 79 & 88 & 78 \\
\hline Causa: nefropatía & $\mathrm{DM}+\mathrm{NEA}$ & $\mathrm{DM}+\mathrm{NEA}$ & ERP & No filiada & $N E A+E R P$ & Tumor renal \\
\hline Tiempo de diálisis (m) & 90.3 & 28.1 & 27.3 & 74.5 & 32.6 & 17.3 \\
\hline iCh & 13 & 13 & 3 & 8 & 10 & 9 \\
\hline $\mathrm{CHA}_{2} \mathrm{DS}_{2}$ VASc & 6 & 5 & 3 & 4 & 4 & 3 \\
\hline HAS-BLED & 6 & 5 & 4 & 6 & 6 & 5 \\
\hline$A C$ por FA & Permanente & Paroxística & Paroxística & Paroxística & Paroxística & Paroxística \\
\hline Tratamiento previo & HBPM + Clop & Clop & Acen & Acen + Clop & Warf & Acen \\
\hline Indicación de COI & $\begin{array}{l}\text { Hemorragia } \\
\text { grave } 0 \\
\text { recurrente }\end{array}$ & INR lábil & $\begin{array}{l}\text { Hemorragia } \\
\text { grave } 0 \\
\text { recurrente }\end{array}$ & $\begin{array}{l}\text { Hemorragia } \\
\text { grave } 0 \\
\text { recurrente }\end{array}$ & $\begin{array}{l}\text { Hemorragia } \\
\text { grave } 0 \\
\text { recurrente }\end{array}$ & $\begin{array}{l}\text { Hemorragia } \\
\text { grave } 0 \\
\text { recurrente }\end{array}$ \\
\hline ETE Control 3-6 m & $\begin{array}{l}\text { Sin fugas ni } \\
\text { trombos en } \\
\text { dispositivo }\end{array}$ & $\begin{array}{l}\text { Sin fugas ni } \\
\text { trombos en } \\
\text { dispositivo }\end{array}$ & $\begin{array}{l}\text { Sin fugas ni } \\
\text { trombos en } \\
\text { dispositivo }\end{array}$ & $\begin{array}{l}\text { Sin fugas ni } \\
\text { trombos en } \\
\text { dispositivo }\end{array}$ & Fuga $<5 \mathrm{~mm}$ & $\begin{array}{l}\text { Sin fugas ni } \\
\text { trombos en } \\
\text { dispositivo }\end{array}$ \\
\hline Tratamiento & Ninguno & AAS & AAS & AAS & AAS & AAS \\
\hline Episodios & $\begin{array}{l}\text { Salida por } \\
\text { sepsis }\end{array}$ & Sin episodios & Sin episodios & Sin episodios & Sin episodios & $\begin{array}{l}\text { Muerte súbita } \\
\text { a los } 12 \\
\text { meses }\end{array}$ \\
\hline
\end{tabular}

DM: diabetes mellitus; NEA: nefroangioesclerosis; ERP: enfermedad renal poliquística; iCh: índice de Charlson; HBPM: heparinas de bajo peso molescular; Clop: clopidogrel; Acen: acenocumarol; Warf: warfarina; COI: cierre de la orejuela izquierda; ETE: eco transesofágico; AAS: ácido acetilsalicílico; AC: anticoagulación; FA: fibrilación auricular.

Tabla 2. Características técnicas del procedimiento

\begin{tabular}{|l|c|}
\hline \multicolumn{2}{|c|}{ Morfología de la orejuela (\%) } \\
\hline $\begin{array}{l}|c| \\
\text { “Ala de pollo" } \\
\text { “Calcetín de viento" }\end{array}$ & $4(66.6)$ \\
\hline Coliflor" & $1(16.7)$ \\
\hline Diámetro máximo de ostium (mm) & $22.2 \pm 1.3$ \\
\hline Diámetro mínimo de ostium (mm) & $17.7 \pm 1.5$ \\
\hline Profundidad (mm) & $23.5 \pm 3.7$ \\
\hline Tamaño del dispositivo (mm) & $26.5 \pm 1.2$ \\
\hline Tiempo de endoscopia (min) & $23.75 \pm 3$ \\
\hline Duración total implante (min) & $75 \pm 38$ \\
\hline Cantidad de contraste (ml) & $72.5 \pm 15$ \\
\hline Éxito en el implante (\%) & $6(100)$ \\
\hline Complicaciones durante el procedimiento (\%) & 0 \\
\hline \multicolumn{1}{|c|}{ Tratamiento tras el implante (\%) } \\
\hline Doble antiagregación
\end{tabular}

Todos los pacientes recibieron el alta en las 24 horas posteriores a la intervención. En la tabla 2 se resumen los principales detalles técnicos.
En la serie de los autores, la tasa de éxito es absoluta $(100 \%)$ y la tasa de complicaciones durante el procedimiento inexistente $(0 \%)$, sin perder de vista que el número de pacientes fue pequeño. Esto objeta la creencia de que los pacientes con ERCT sometidos a procedimientos cardiológicos intervencionistas tienen mayor riesgo de complicaciones, debido a que son sujetos más frágiles que la población general y con más cantidad de comorbilidades 5 . Chak, et al. comunicaron una serie de 196 pacientes sometidos a $\mathrm{COI}$ en la que se compararon dos grupos, con y sin ERC, encontrándose una mayor tasa de complicaciones periprocedimiento (9.9\% vs. $2.4 \%, p=0.04)$ en el grupo con deterioro de la función renal, a expensas del taponamiento cardiaco $(8.5 \% \text { vs. } 0.8 \%, p=0.01)^{6}$. Genovesi, et al., publicaron en fecha reciente datos de la mayor serie informada hasta el momento y demostraron en 50 pacientes que, a pesar de la edad avanzada y las múltiples comorbilidades que sufren los pacientes con ERCT, el implante del dispositivo es factible y seguro, con una tasa elevada de éxito $(100 \%)$ y una tasa baja de complicaciones durante y después del procedimiento (sólo se describieron tres complicaciones menores ${ }^{7}$ ). Kefer, et al., por su 
parte, publicaron una serie en la que se analiza el efecto que la ERC tiene en la prevención del ictus en pacientes sometidos a COI; en esta serie se consideró a pacientes con diversos grados de ERC, incluidos aquéllos con ERCT (estadio V). En ellos, la tasa de éxito en el procedimiento fue también alta (> 98\%) y la de complicaciones baja $(5.1 \%)$, sin observar diferencias entre los pacientes con deterioro de la función renal y sin ella, ni tampoco entre los que mostraban deterioro leve o $\mathrm{ERCT}^{8}$. El mayor conocimiento sobre la anatomía de la Ol, el uso racional de las técnicas de imagen, la mayor experiencia acumulada en el implante de este tipo de dispositivos, así como los programas de proctorización que facilitan los fabricantes, hacen que este tipo de procedimientos sea algo seguro a pesar de que la unidad de hemodinámica se encuentre en la "curva de aprendizaje", como es este caso.

Durante una mediana de seguimiento de 272 días, y con controles con ecocardiografía transesofágica (ETE) a los tres, seis y 12 meses, no se han encontrado trombos relacionados con el dispositivo en ningún caso, y tan sólo una pequeña fuga en uno de ellos que no requirió intervención por ser menor de $5 \mathrm{~mm}$. De la misma manera, hasta la fecha no se ha registrado ningún episodio de tipo cardioembólico ni sangrado significativo $($ BARC > 2).

A pesar de que la mortalidad relacionada con el procedimiento ha sido nula, dos pacientes han fallecido, uno por sepsis y otro por muerte súbita; si bien no se puede descartar que ésta fuera de causa embólica, lo más probable es que se debiera a la cardiopatía avanzada subyacente que presentaba el paciente.

La complejidad clínica de estos pacientes es muy alta y se ha tratado de medir con el índice de comorbilidad de Charlson (iCh), no tanto para evaluar a los pacientes sino para compararlos con los de otras series en el futuro. Se ha recurrido al iCh debido a que se ha empleado de forma amplia como variable de ajuste en distintos modelos pronósticos ${ }^{9}$. El iCh de la serie de pacientes de los autores es muy alto (media de 9.3): los dos sujetos fallecidos eran de los más altos (13 y 9). Es posible que en el futuro este índice pueda ayudar a seleccionar, dentro de esta población de pacientes, a aquellos que pueden beneficiarse más de esta medida preventiva a medio y largo plazos.

Aunque esta serie incluye a un número reducido de pacientes y no se pueden inferir conclusiones estadísticas, las perspectivas de la técnica son muy promisorias. Sin embargo, los estudios clínicos Left atrial appendage occlusion vs. usual care in patients with atrial fibrillation and severe chronic kidney disease (WatchAFIB) y el The strategy to prevent hemorrhage associated with anticoagulation in renal disease management (STOP HARM) trial se detuvieron a finales de 2018 por problemas en el reclutamiento de pacientes. En la actualidad está en marcha el Registro de cierre percutáneo de la orejuela izquierda con dispositivo Watchman ${ }^{\circledR}$ en pacientes con fibrilación auricular no valvular y enfermedad renal crónica en hemodiálisis (NCT NCT03446794), un registro multicéntrico español que cuenta con un objetivo combinado a los 24 meses de ictus o accidente isquémico transitorio, sangrado BARC-2 y embolia sistémica, así como objetivos secundarios de seguridad relacionados con el procedimiento y el seguimiento ecocardiográfico.

Es evidente que el nefrólogo es reticente en este momento a usar una técnica invasiva como medida preventiva, aunque ello suponga retirar la ACO a los pacientes y sus beneficios conocidos, y también el cardiólogo que es reacio a priori a tratar a pacientes con una comorbilidad considerable. Por lo tanto, los autores destacan la necesidad de establecer vías de comunicación entre ambas especialidades, que permitan una correcta selección de los pacientes y optimizar así el beneficio que esta técnica ofrece.

\section{Conflicto de intereses}

Ninguno.

\section{Financiamiento}

Ninguno.

\section{Responsabilidades éticas}

Protección de personas y animales. Los autores declaran que para esta investigación no se han realizado experimentos en seres humanos ni en animales.

Confidencialidad de los datos. Los autores declaran que han seguido los protocolos de su centro de trabajo sobre la publicación de datos de pacientes.

Derecho a la privacidad y consentimiento informado. Los autores declaran que en este artículo no aparecen datos de pacientes.

\section{Bibliografía}

\footnotetext{
1. Olesen J, Lip G, Kamper AL. Stroke and bleeding in atrial fibrillation with chronic kidney disease. N Engl J Med. 2012;16:625-635.
} 
2. Brodsky SV, Nadasdy T, Rovin BH, Satoskar AA, Nadasdy GM, Wu HM, et al. Warfarin-related nephropathy occurs in patients with and withou chronic kidney disease and is associated with an increased mortality rate. Kidney Int. 2011;80(2):181-9.

3. Cozzolino M, Brancaccio D, Gallieni M, Slatopolsky E. Pathogenesis of vascular calcification in chronic kidney disease. Kidney Int. 2005;68(2) 429-36.

4. Ruff CT, Giugliano RP, Braunwald E, Hoffman EB, Deenadayalu N, Ezekowitz MD, et al. Comparison of the efficacy and safety of new oral anticoagulants with warfarin in patients with atrial fibrillation: a meta-analysis of randomised trials. Lancet. 2014;383(9921):955-62.

5. Polyzos K, Konstantelias A, Falagas M. Risk factors for cardiac implantable electronic device infection: a systematic review and meta-analysis. Europace. 2015;17(5):767-777.
6. Chak YS, Cheung GSH, Chan CKY, Kam KKH, Chan AKY, Au SSW, et al. Effect of chronic kidney disease on left atrial appendage occlusion outcome: a single-centre retrospective analysis. JACC. 2018;71:A302.

7. Genovesi S, Slaviero G, Porcu L, Casu G, Bertoli S, Sagone A, et al Implant success and safety of left atrial appendage occlusion in end stage renal disease patients: peri-procedural outcomes from an Italian dialysis population. Int $\mathrm{J}$ Cardiol. 2018;262:38-42.

8. Kefer J, Tzikas A, Freixa X, Shakir S, Gafoor S, Nielsen-Kudsk JE, et al. Impact of chronic kidney disease on left atrial appendage occlusion for stroke prevention in patients with atrial fibrillation. Int $\mathrm{J}$ Cardiol. 2016;15(207):335-340.

9. De Groot V, Beckerman H, Lankhorst GJ, Bouter LM. How to measure comorbidity. A critical review of available methods. J Clin Epidemiol. 2003;56:221-9. 